\title{
Coordinated Collaboration between Heterogeneous Distributed Energy Resources
}

\author{
Shahin Abdollahy, ${ }^{1}$ Olga Lavrova, ${ }^{1}$ and Andrea Mammoli ${ }^{2}$ \\ ${ }^{1}$ Electrical and Computer Engineering Department, University of New Mexico, Albuquerque, NM 87131, USA \\ ${ }^{2}$ Mechanical Engineering Department, University of New Mexico, Albuquerque, NM 87131, USA \\ Correspondence should be addressed to Shahin Abdollahy; shahinab@unm.edu
}

Received 31 July 2014; Revised 5 November 2014; Accepted 13 November 2014; Published 2 December 2014

Academic Editor: Guillaume Zoppi

Copyright (c) 2014 Shahin Abdollahy et al. This is an open access article distributed under the Creative Commons Attribution License, which permits unrestricted use, distribution, and reproduction in any medium, provided the original work is properly cited.

\begin{abstract}
A power distribution feeder, where a heterogeneous set of distributed energy resources is deployed, is examined by simulation. The energy resources include PV, battery storage, natural gas GenSet, fuel cells, and active thermal storage for commercial buildings. The resource scenario considered is one that may exist in a not too distant future. Two cases of interaction between different resources are examined. One interaction involves a GenSet used to partially offset the duty cycle of a smoothing battery connected to a large PV system. The other example involves the coordination of twenty thermal storage devices, each associated with a commercial building. Storage devices are intended to provide maximum benefit to the building, but it is shown that this can have a deleterious effect on the overall system, unless the action of the individual storage devices is coordinated. A network based approach is also introduced to calculate some type of effectiveness metric to all available resources which take part in coordinated operation. The main finding is that it is possible to achieve synergy between DERs on a system; however this required a unified strategy to coordinate the action of all devices in a decentralized way.
\end{abstract}

\section{Introduction}

As the electric utility industry enters an increasingly competitive environment, utilities must concern themselves with the market value of the services they provide and the cost of providing those services. At the same time utilities are still burdened with the obligation to serve their customers with adequate reliability. Utilities must undertake new investments in demand-side resources to meet this obligation [1].

Distributed energy resources (DERs) have demonstrated potential advantages to address the challenges utilities are facing. Photovoltaic generation (PV), fuel cell (FC), battery energy storage (BES), natural gas powered GenSet (NGPG), wind turbine, thermal storage (TS), and micro-CHP (combined heat and power) are exemplary DERs technologies successfully deployed at power distribution level. On the other hand, in light of increased needs for both energy efficiency and high reliability the microgrids are gaining increasing interest. A microgrid is a collection of DERs that from the viewpoint of the utility is controllable, acts as a single load, and is able to function in both grid-tied and islanded modes [2].

Recent trends in small-scale distributed generation particularly drastic price reductions of PV and small wind turbines will soon result in high penetration levels of variable generation, some of which are not directly controlled by the utility. Moreover, much of this generation is highly intermittent. As a result, concerns are growing on the ability of the utility to maintain uniform power quality over the length of a distribution feeder. Recent work has demonstrated that BES system can effectively perform two important tasks [3]:

(i) shifting renewable power delivery to times of peak demand,

(ii) smoothing intermittent PV generation before it is injected into the feeder.

BES systems could be utilized to offset the variability of the power, supplied by renewable energy resources as a power-balancing resource [4]. On the other hand, BES 
system can provide a dispatchable resource in conjunction with nondispatchable renewable resources by providing an energy-balancing service [5]. Power-balancing service, in this context, refers to the services provided by the corresponding resources to compensate the instantaneous power deficit or surplus which otherwise could cause flicker or overvoltage issues. In general, a provider of such a service is characterized by high power and low energy capacity. Contrarily, energy-balancing is associated with long term bulk energy compensation. An energy-balancing service provider has a considerably high energy capacity with limited ramping capability. According to [6], the promising advantages of distributed storage systems will be the following:

(i) demand peak shaving at substations,

(ii) smoothing for high PV penetration,

(iii) ancillary services,

(iv) improving transmission and distribution reliability,

(v) generation/demand balancing.

Thermal storage is another type of DERs receiving renewed attention [7]. In the commercial and residential building sector, cooling loads account for much of the peak demand (approximately 40\%) [8]. Cold storage devices allow the cooling load to be shifted to periods of lower demand. While cold storage devices are too small to affect the overall system demand when acting individually, their aggregated action could be significant [9].

A modern hybrid distribution system in this context refers to a distribution feeder which incorporates coordinated loads, renewable energy resources (mostly small-scale PVs), coordinated BES systems, and the underlying communication and control infrastructure. The system may include more than one type of renewable energy resource and BES system. There are various energy storage systems available commercially that vary in applicability, type, and cost which could be utilized in the so-called smart grid.

This paper is organized in four sections. Section 1 is this introduction and covers some existing literatures related to the subject matter. Section 2 introduces the distribution feeder under study and considers the development of models capable of performing various time-series analyses. PV penetration, spatial distribution effects, and multiagent operation are also studied. Section 3 covers some simulations performed to demonstrate the potentials of a coordinated set of distributed energy resources and their benefits to the power system. Section 4 offers some conclusions and extensions.

\section{System Description and Preliminary Analysis}

A DERs demonstration project located at Studio 14 (ST14) distribution feeder in Albuquerque, New Mexico [10], combines a microgrid-consisting of a $240 \mathrm{~kW}$ natural gas powered generator (NGPG), an $80 \mathrm{~kW}$ phosphoric acid fuel cell, a $50 \mathrm{~kW}$ PV field, a lead acid battery storage system, and a hot and cold thermal storage system-with a $500 \mathrm{~kW}$ utility scale PV array and an associated $1 \mathrm{MWh}$ shifting and

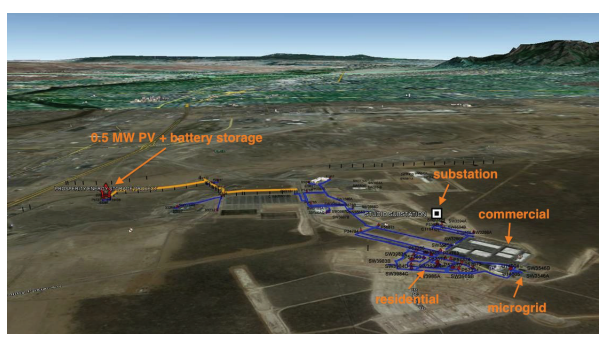

FIgURE 1: Studio 14 distribution feeder in Albuquerque, NM, hosting a number of DERs demonstration projects that could interact to maximize economic value and system performance.

smoothing battery bank. Also on the same distribution feeder are several businesses (including movie studios with large ice storage devices) as well as a growing residential PV-ready community, which when fully built has the potential to reach a high penetration. An aerial image of the feeder is shown in Figure 1.

GridLAB-D is a grid simulator, developed by Pacific Northwest National Laboratory (PNNL) [11]. It is designed specifically for modeling complex distribution systems with distributed resources and provides various functions to model smart grids. A complete model of the feeder is built in GridLAB-D based on the data from PNM's Geographic Information System (GIS). The model is tested and validated against the historical demand and monthly consumption data and is reported in a separate paper [12].

2.1. High Penetration Distributed PV. A partly cloudy day in early fall is selected for high PV penetration study. Historical data show that the feeder load is lower in early fall or late spring than in hot summer. On the other hand, the PV generation power is not far less than its peak. Therefore, the $\mathrm{PV}$ penetration, defined as the ratio between the peak PV power to peak load, would be close to its highest value in early fall or late spring. Any partially cloudy day in early summer or late spring may also be considered for high penetration studies.

Power distribution systems will soon be facing increased number of PV installations in the form of roof top smallscale PV systems. To modify the developed model of the distribution feeder ST14 and to study the high PV penetration case, the total number of PV systems and subsequently the peak generation power were assumed to grow 5-fold. The prospective PV systems were randomly distributed along the feeder.

No significant change in the voltage profile was observed for most nodes except those ones connected to the end of weak laterals. Even highly fluctuating PV generation did not make noticeable difference in feeder voltage profile. Results conform with the study on high PV penetration in [13] which shows that distributed PV systems installed close to the main body of the feeder do not cause a significant voltage fluctuation. However, remote nodes connected to long and weak feeder laterals may suffer from voltage flicker, sag, and swell due to fluctuations in PV generation power. 
2.2. Spatial Distribution of the PV Generators. Traditionally, a distribution system design is based on unilateral flow of energy from the substation into the points where it is consumed. The mechanisms responsible for controlling the power quality could not efficiently operate in existence of excessive variations in voltage and load and also bidirectional energy flow. Voltage correcting devices such as tap changers and VAR compensators do not usually respond to highfrequency changes effectively. Therefore by incorporating large capacities of renewable energy (RE) resources in the distribution system, utilities face critical challenges to maintain power quality and continuity of service in presence of fluctuating power generation caused by passing clouds at very high/very low wind speeds [14].

Spatial diversity is an inherent consequence of a semiuniform integration of a large number of RE resources in a distribution feeder. Higher frequency components of the total generated power are eliminated as a result of such diversity. That is studied by adding PV to a number of random nodes to achieve a desired PV penetration level, assuming that highaltitude (cloud-level) wind blows at maximum $60 \mathrm{mph}$ from north to south and moves the clouds in that direction, which, respectively, causes sharp fluctuations in the PV output power. When the wind blows at lower speeds, the expected ramping rate at the PV output is minimal. Therefore, only the worst case (high speed) is studied. At the assumed wind speed, it takes at least $T_{\min }$ (6 minutes in this case) for the same cloud pattern to move from the northernmost corner to the southernmost corner of the feeder area. To model cloud coverage at node $i$, the benchmark PV output is shifted by a random time delay uniformly distributed between zero and $T i_{\min }$. The benchmark node is the only node in which the PV output is recorded. $T i_{\min }$ is the estimated time taken by the cloud to travel from the benchmark node to node $i$. Solar irradiance level is assumed constant during that time period.

To understand the differences in spectral concentration of the PV energy between a distributed PV system and an equally sized centralized PV plant, a large PV generator is modeled. The large aggregated PV system (APVS) has a generation capacity equal to the total cumulative power of the distributed PVs connected to the same feeder. The APVS is considered spatially centralized which means all PV cells are subjected to the same solar irradiance at any point in time. Spectral analysis of the output power in a 24-hour period reveals that spatial distribution of the PV systems over the feeder area reduces the high-frequency contents of the power spectrum significantly. In Figure 2, the spectral density function which is defined as the cumulative density function (CDF) of the fast Fourier transform (FFT) of the output power is shown for two cases: distributed PV and APVS. The smoothing effect thanks to the spatial diversity of the PV system could be understood by comparing the frequencies below which $95 \%$ of the total energy of the associated signal is concentrated. The $95 \%$ point in distributed PV case is almost a tenth of its value for APVS. That means that the higher frequency contents of the PV power due to partial cloud coverage are filtered out as a result of distributing PV generators. Also a large portion of the delivered energy is concentrated in the lower frequency range. Therefore, by

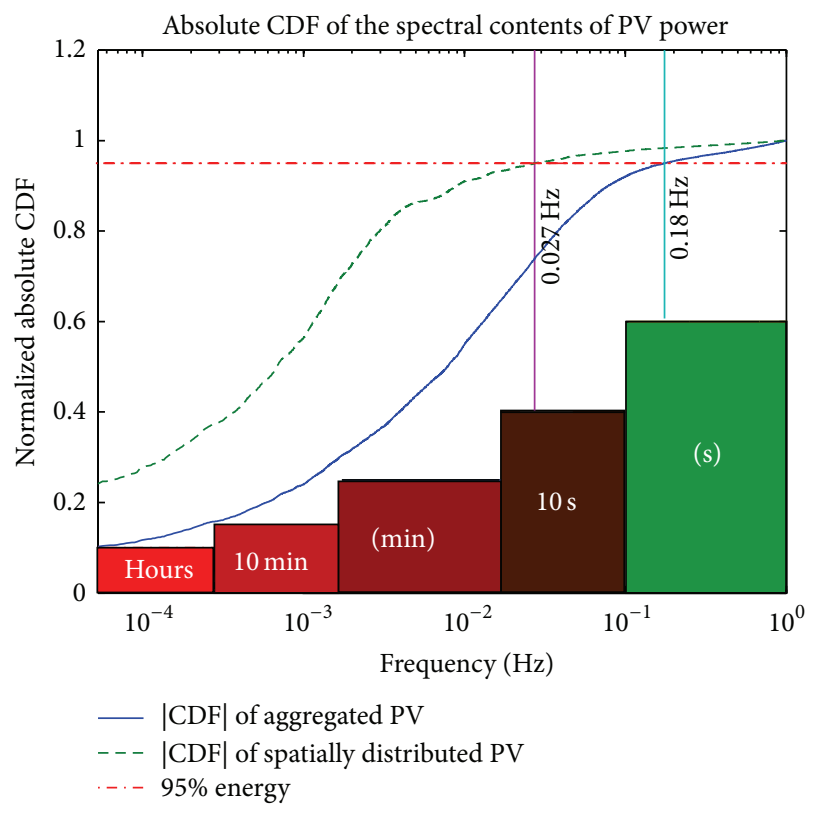

FIGURE 2: Normalized absolute density of the spectral contents of the generated power for distributed PVs and the equivalent aggregated PV (data from PNM).

distributing the energy resources (in this case PV systems) over the service area, necessity of any BES system to mitigate the PV output fluctuations would be eliminated. On the contrary, it is the PNM demonstration project which is comprised of a PV farm and a $500 \mathrm{~kW}$ smoothing battery designed to mitigate the high-frequency PV power variations.

More accurate analysis by incorporating exact coordinates, availability information, and nominal rating of each individual PV could be carried on if desired. However, a steady and uniform growth in installation of new PV systems transforms the distribution feeders to spatially diverse and coherent collections of DERs. In such feeders the assumption of uniform distributions for the DERs would not deviate significantly from reality and consequently the simulations presented in this section would be highly accurate.

2.3. Multiagent Operation. Widespread usage of the DERs at feeder level makes the capacity of the equivalent aggregated resource large enough to be comparable to the total feeder's demand, which, by raising the complexity of the system, makes the coordination between the agents and the grid operator maintain power quality and continuity of service more crucial and challenging. On the other hand by utilizing a well-diversified set of DERs in the distribution system, it would be less inaccurate to build a simplified system model which incorporates a uniform distribution of DERs. One ultimate goal would be to dispatch the available distributed capacity in the same way as a conventional resource would be dispatched. Coordination is the key solution to provide such capability to form a dispatchable resource which could be leveraged to maintain the balance between the load and the supply in a microgrid or feeder. In a more complicated 
form, coordination could be applied toward fulfillment of a cost minimization or benefit maximization solution.

A good example of coordination between the agents was the PNM Prosperity PV plus storage project in Albuquerque, NM. As described previously, Prosperity was connected to the ST14 feeder. The feeder model was developed and validated against the recorded information. Several studies have been conducted on the case and have been reported [15]. In terms of the number of agents and complexity of communication, Prosperity was a rather straightforward case because all the agents (the PV farm, the fast smoothing battery, and the slow shifting battery) were at the same location. Therefore, the necessary data were transparent to the controllable agents (the smoothing battery and the shifting battery) almost instantaneously. However, in terms of capacity of the storage system and the developed power shifting and smoothing algorithms, it has been a rather successful demonstration of a coordinated multiagent system. The following collective goals were achieved by implementing some sort of coordination between the agents:

(i) power quality which consists in the following:

(a) smoothing the high-frequency portion of the PV generation,

(b) preventing possible overvoltage due to excessive PV generation during nonpeak demand time,

(c) maintaining the feeder voltage stability;

(ii) operations which consist in the following:

(a) arbitrage,

(b) firming and shaping,

(c) shifting.

Arbitrage refers to taking profit by purchasing and storing electricity when it is cheap and selling it back to the grid when profitable. This operation is based on dynamic pricing of electricity. Firming and shaping are the utility nomenclature referring to transforming the intermittent and unpredictable renewable energy resources like PV and wind into a dispatchable resource. Shifting means storing energy when not needed and releasing it when demanded. This technology can help utilities reduce their need for cycling peak generation units which are costlier than base generation.

Coordination between the agents would not be possible without controllability. Resources with a renewable origin such as PV or wind are among the uncontrollable types. However, by incorporating a balanced set of controllable and intermittent resources and by implementing appropriate coordination methods, it would be possible to attain a collective goal at the power distribution level.

To devise a coordination method for controlling the distributed agents and reaching a common objective, one must first consider the types and the capacities of the available agents (DERs). Different resources are mainly characterized by their capacity, controllability/intermittency, effectiveness, cost of operation, and maintenance and availability; some attributes are relatively easier to evaluate than others. For instance, capacity is a measurable parameter, but effectiveness

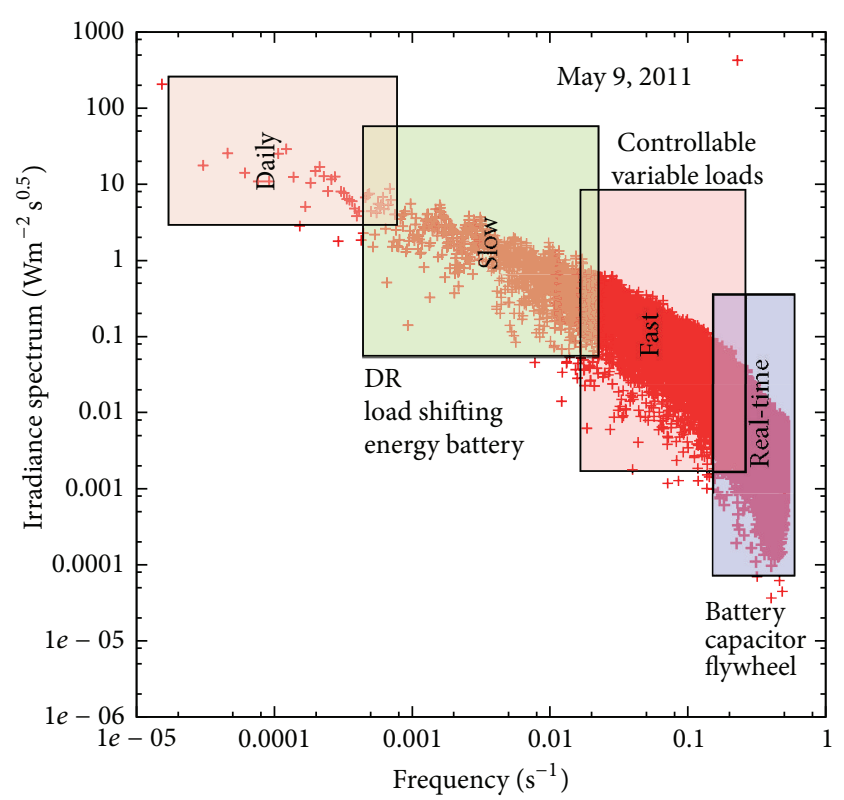

FIGURE 3: PV power spectral contents in a partly cloudy day. The spectrum is divided into frequency ranges based on the amount of energy contents (data from PNM).

is a complex attribute. Definition of effectiveness is highly depending on the collective goal to be reached. As an example, local PV generation at a remote node is considered a noneffective parameter in controlling a specific node's voltage. In such a case, effectiveness is inversely related to the electrical distance between the two nodes.

For instance, the intermittency characteristics of the PV power can be examined using power spectral density plots [16]. As an example, the spectral contents of a highly intermittent day in May are displayed in Figure 3 and were collected from the $500 \mathrm{~kW}$ PV array irradiance sensors described the beginning of this paper. By dividing the spectral contents into separate subsections, different techniques for managing those frequencies can be studied for each section.

The power spectral density plot reveals that highfrequency fluctuations carry little power (and as a consequence little energy). Thus, these can be compensated with fast-responding devices (e.g., capacitors, flywheels, and some high-performance batteries) with high power/ramping capacity and relatively small energy capacity. At the other end of the scale, daily solar power generation cycles can, at least partially, be compensated with thermal storage devices, offpeak (base load) grid generation, and night-time grid-scale wind generation when available.

\section{Simulations}

Simulations were conducted using GridLAB-D, an opensource agent-based modeling tool which allows the entities (agents) within the model to interact with each other and with their external environment.

Several cases were studied to demonstrate potential benefits of coordination between distributed agents for the utility 
or the microgrid operator in achieving a collective goal. It was assumed that the required data for every agent would be available instantaneously and at no cost. Such assumption helped to keep the control scheme less complex. Additional cost and delay for data acquisition were later taken into account in finding solutions for reducing or eliminating the need for data transmission and the unavoidable delay.

3.1. Partial Smoothing. The medium and high-frequency fluctuations of the PV power can potentially deteriorate the quality of power delivered to the customers. Smoothing is a familiar phenomenon introduced to mitigate the fluctuations induced by the energy resources with uncontrollable nature. The capabilities of the Prosperity BES system in smoothing the intermittency of the PV output are successfully demonstrated in the past. Simply put, the smoothing algorithm constantly generates a power reference signal for the smoothing battery which correlates with the difference between the moving average of the PV output and its instantaneous value. The smoothing process is a costly operation since it incorporates frequent charging/discharging cycles of the costly smoothing battery. Due to practical limitations, the number of charging and discharging cycles is limited. Should there be a less costly solution for the same purpose, it would be plausible to utilize the BES system in a more efficient or more profitable application and offset the costs by using a cheaper resource where possible.

Considering that the operating costs of the natural gas GenSet in terms of $\$ / \mathrm{kW}$ and $\$ / \mathrm{kWh}$ were less than those of the BES system, a microgrid consisting of a PV farm, a BES system, and a GenSet was set up to study how a less expensive resource (GenSet) could offset a costlier resource without compromising performance.

The natural gas GenSet is forced to follow the ramp rate of the injected PV power in an opposite direction. Two methods for calculating the power reference signal are defined: ramp rate compensation (RRC) and moving average compensation (MAC). In the former, the reference signal is generated by multiplying the sampling time by the scaled ramp rate of the PV output and then by adding to $P_{\text {nom }} / 2$ (1). In the latter, the difference between the moving average and the instantaneous value of the PV output is calculated, scaled, and then added to the instantaneous power of the GenSet (2):

$$
\begin{gathered}
P_{\mathrm{ref}_{\mathrm{RR}}}=T_{s} \cdot k_{1} \cdot \mathrm{PV}_{\mathrm{RR}}+\frac{P_{\text {nom }}}{2}+k_{2} \cdot \Delta P+k_{3} \cdot \Delta E, \\
P_{\mathrm{ref}_{\mathrm{MA}}}=P_{\text {inst }}+k_{4} \cdot P_{\text {diff }}+k_{5} \cdot \Delta P+k_{6} \cdot \Delta E,
\end{gathered}
$$

where $T_{s}$ refers to the sampling time, $k_{1} \cdot \mathrm{PV}_{\mathrm{RR}}$ refers to the weighted ramp rate of $\mathrm{PV}$ output, $k_{2}, k_{4} \cdot \Delta P$ refers to the power balancing loop, $k_{3}, k_{5} \cdot \Delta E$ refers to the energy balancing loop, and $k_{4} \cdot P_{\text {diff }}$ refers to the weighted difference between M.A. and instant output.

Although the GenSet is a net positive energy resource, it behaves in the same way a combination of a fixed power source $\left(\right.$ Power $\left.=P_{\text {nom }} / 2\right)$ and a battery operates. Figure 4 illustrates how the GenSet has contributed to the smoothing of the aggregated output of the system. The output power of the BES in the presence and in the absence of the

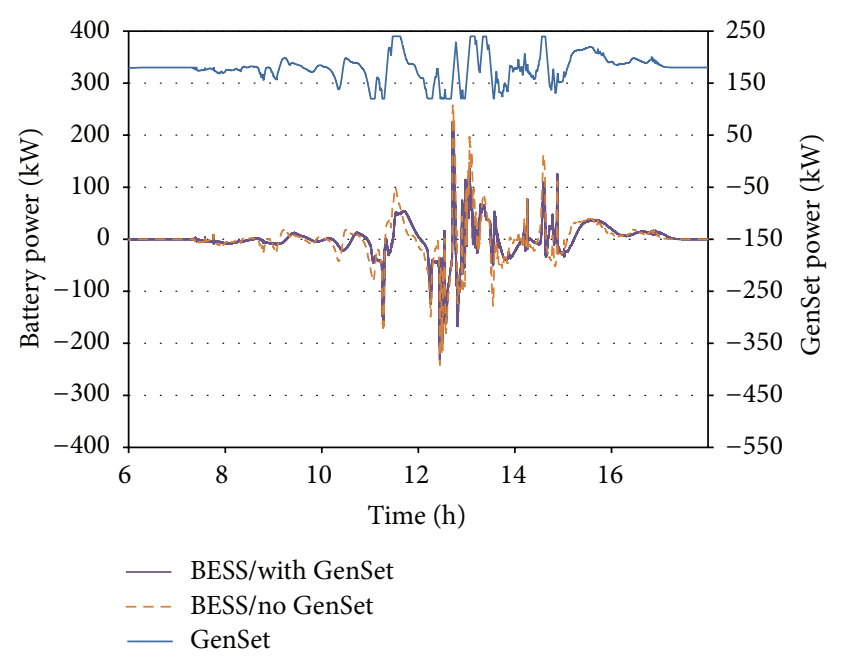

FIGURE 4: BES system power exchange diagrams with and without GenSet contribution are shown. Peak BES system power is reduced in presence of GenSet.

GenSet is also shown. Although the GenSet has less ramp rate capacity $(0.3 \mathrm{~kW} / \mathrm{sec})$ compared to the BES smoothing battery $(50 \mathrm{~kW} / \mathrm{sec})$, to some extent it has moderated the contribution of the BES in smoothing. Assuming that in general a gas powered GenSet is relatively less costly to operate than a BES, the coordinated system has a lower cost of operation by utilizing a second resource for energy.

The PV output power, its moving average (sliding window size: $1000 \mathrm{sec}$ ), and the total system output are shown in Figure 5. There is a direct correlation between the level of smoothness of the total output and the amount of power exchanged between the resources (BES system and GenSet in the studied case) and the grid. That means that there is always a tradeoff between the cost associated with smoothing and the output quality. A ranking mechanism should be employed to designate which resource and for how much it should contribute to the common goal. A simple solution could involve cost and electrical distance. In the above example, the GenSet had $100 \%$ weight and higher priority. That is why the GenSet was set to operate with full capacity and the BES had to compensate for the balance of the power required for smoothing.

The same concept could be applied to any combination of DERs and demand side management methods (demandresponse is considered a resource).

3.2. Peak Shaving and Demand Shifting. At the lower end of the frequency spectrum, daily scheduling of thermal storage devices can result in reduced feeder load peaks (peak shaving). It is known that cooling loads account for a large portion of the peaking loads in the summer, and they have been increasing due to population shifts to warmer climates. However, because optimization of thermal storage device schedules is usually strictly local to the facility, it is possible that the collective action of many thermal storage sites produces artificial peaks, for example, if all storages charge at the time of minimum energy cost. For this study, 


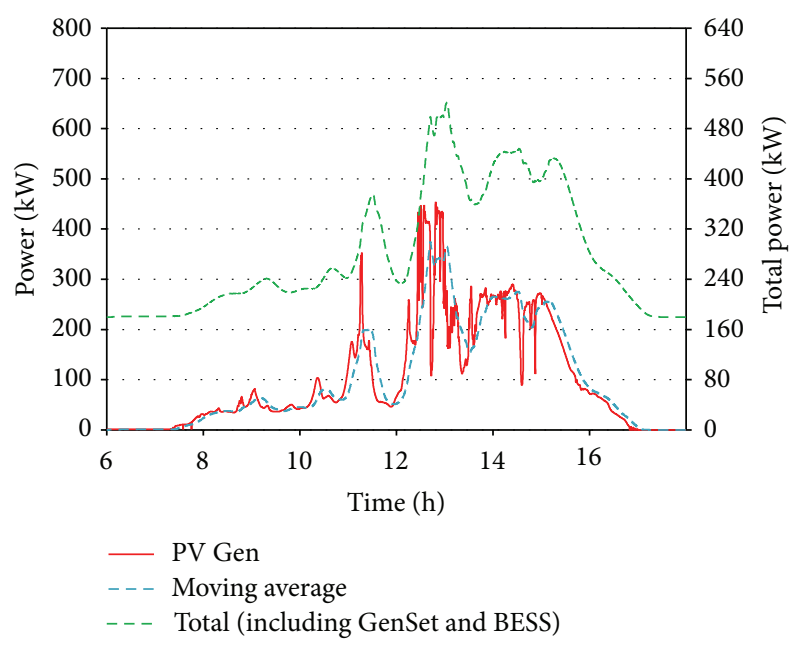

FIGURE 5: Total generated power is smoother than PV output thanks to the contributions from BES system and GenSet.

20 commercial buildings are added to the feeder model. Each building's load is separated into cooling and noncooling loads. In turn, the cooling load can be served either by depleting the ice storage or by conventional cooling systems.

To accommodate redundant cooling in a building with thermal storage, the operator requires deciding how the cooling is to be provided. The simplistic solution is to fully charge the storage off-peak and to deplete it on-peak. However, a full charge is not always necessary, especially in shoulder seasons (moderately hot), and in fact could lead to additional thermal losses. Moreover, the period of maximum discharge could be selected so that the maximum power demand is minimized. Optimization of such parameters can be performed effectively, for example, by using Software-as-a-Service, as in the case of the Distributed Energy Resources-Customer Adoption Model (DER-CAM) developed by Lawrence Berkeley National Laboratory [17]. However, from a system's perspective, optimization of the individual site based only on information at the site itself could be less than optimal globally. For example, in a system such as the feeder described here, peak solar generation could be used directly to supply loads, while peak load could be shaved by thermal storage, as an alternative to storing the PV peak in a battery, to deliver it later to serve cooling loads. Local optimization would not see this opportunity, since the PV generation is not colocated with the thermal storage. Another possible downside of local optimization is the possibility of many thermal storage devices beginning to charge at the same time, to take advantage of the off-peak part of the rates. This could create an artificial peak, as well as a very high ramp rate that would be difficult to manage using conventional devices such as LTCs.

The conflict between local optimization and global optimization is difficult to resolve. On the one hand, a local optimization only considers benefits to the end user. Because of the small number of parameters involved, it is a tractable problem, which can be solved quickly on commonly available hardware, by any available service. On the other hand, global

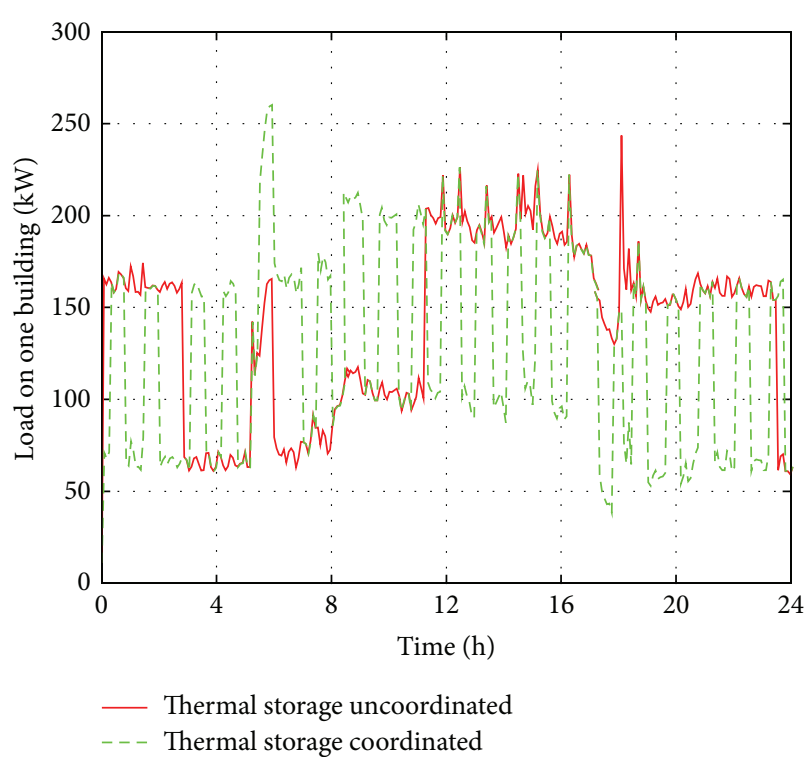

Figure 6: Operation of thermal storage with uncoordinated and coordinated storage. Note that coordinated storage cycles frequently, while uncoordinated storage generally cycles only twice per day, offpeak (charging) and on-peak (discharging).

optimization would involve substantially higher computation costs, and the optimization provider would need to be the same for all end-users (e.g., the utility itself). One way to provide some feedback from the global system to the local optimization would be to provide some form of "real-time" pricing, for example, one based on historical trends for similar weather conditions.

Another possibility is to use statistical techniques to distribute the load according to existing conditions at the feeder. For example, a signal based on the deviation of the feeder load from a desired "optimal" load could be sent to individual loads. The signal could be in the form of a probability of a cooling device "on" status. For example, if the feeder load is higher than the desired value, the probability signal would be reduced, while if the load is low, the probability signal would be high. The individual devices would then act according to the probability, with appropriate hysteresis to prevent excessive cycling. The operation of a storage device for an individual facility for both control options is shown in Figure 6. The load profile for coordinated storage shows frequent changes in load, as a function of the storage device activating and deactivating. While this is not useful (and may even produce slight increases in maintenance costs due to more frequent start-stops) for individual facilities, the positive effects are evident when examining the aggregate load due to commercial facilities.

In Figure 7, commercial load which consists of 20 closely located buildings for the case with no thermal storage is compared with the load resulting from uncoordinated thermal storage and from statistically coordinated storage. The risks resulting from uncoordinated thermal storage are clearly seen, namely, a very sharp increase in power, briefly producing a peak higher than the peak for the case with 


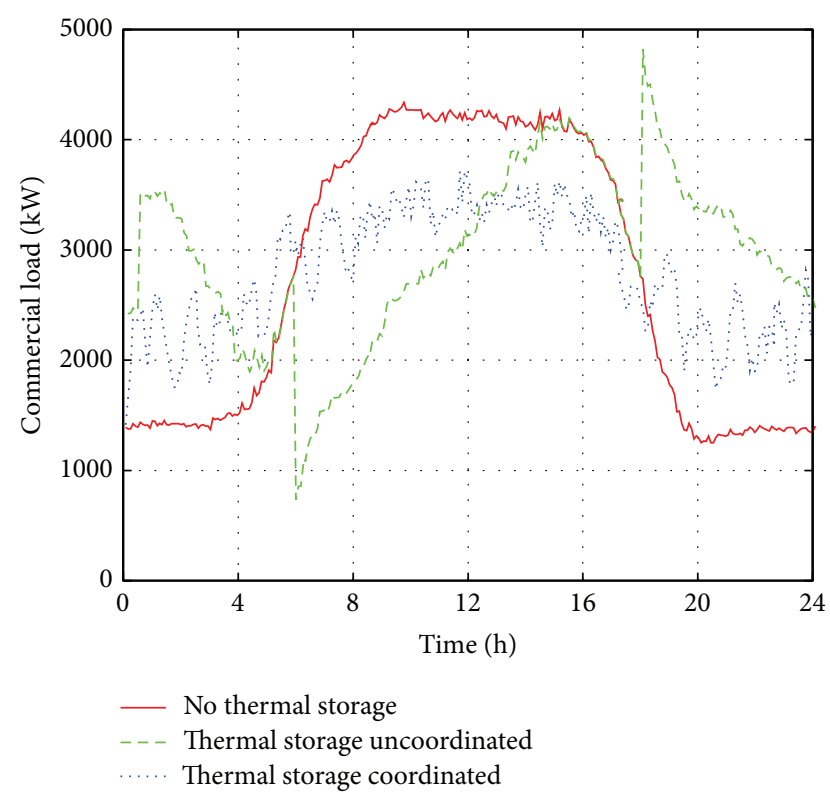

FIgURE 7: Effect of thermal storage on total load due to buildings. The base scenario of no thermal storage is compared with a scenario where thermal storages act in an uncoordinated fashion and the case where stochastic techniques are used to reduce peak loads.

no thermal storage, occurring at the onset of the off-peak period, and a similar drop in power at the end of the off-peak period. The intermittent operation of the storage system due to frequent charge/discharge cycles during the low/high electricity demand periods deteriorates the average capacity of the storage system as opposed to continuous charge/discharge operation. To account for the reduction in the time-averaged capacity of the charging device within the optimization algorithm, the assumed cooling power of the device would be derated based on historical data. Thus, the optimization algorithm would remain local, while allowing for global power management requirements.

\section{Conclusion}

It is known that DERs systems do not necessarily have similar characteristics in terms of capacity, ramping capabilities, cost of operation, availability, controllability, and so on. To define an effective coordination strategy to fulfill the target cost function, available DERs systems must first be assigned a grade or rank which could be interpreted as a weight factor to be used later for contribution adjustments. Of possible ranking strategies, the following are deemed to be more effective for that purpose.

(1) Frequency Band. A BES system can effectively perform two important tasks: to shift the locally generated off-peak power to the time of peak demand and to smooth the intermittent PV power. While this approach demonstrably works, batteries are still very expensive, and their lifetime is limited. One type of DERs that is gaining increasing interest is the microgrid, in light of increased needs for both energy efficiency and high reliability. An islanded microgrid must have enough storage to compensate for any mismatch between load and generation while islanded. Use of thermal storage, as a second type of DERs that is receiving renewed attention, was then extensively examined. Since, in the commercial and residential building sector, cooling loads account for much of the peak demand and cold storage devices allow the cooling load to be shifted to periods of lower demand. While cold storage devices are too small to affect the overall system demand when acting individually, their aggregated action could be significant. By dividing the spectral contents into separate subsections, different techniques for managing those frequencies can be studied for each section. The power spectral density plot reveals that high-frequency fluctuations carry little power (and as a consequence little energy). Thus, these can be compensated with fast-responding devices (e.g., capacitors, flywheels, and some high-performance batteries) with high power/ramp rate capacity and relatively small energy capacity. At the other end of the scale, daily solar power generation cycles can, at least partially, be compensated with thermal storage devices, off-peak (base load) grid generation, and night-time gridscale wind generation when available.

(2) Electrical Distance. In [18] it is shown that the power networks equations can be formed in the bus frame of reference in which the performance is described by $n$ linear independent equations for $n+1$ number of nodes. In the admittance forms, the performance equation can be described by Kirchoff's Laws as

$$
\overline{I_{B}}=\overline{Y_{B}} \cdot \overline{V_{B}}
$$

where

$$
Y_{k l}^{\text {bus }}= \begin{cases}G_{k l}+j B_{k l}, & k \neq l, \\ -\sum_{k \neq l}\left(G_{k l}+j B_{k l}\right), & k=l .\end{cases}
$$

Such definition of the $Y_{\text {bus }}$ matrix captures both the real and reactive portions of the line admittances. In this analysis the inverse of the $Y_{\text {bus }}$ matrix or $Z_{\text {bus }}$ is used which is a nonsparse (dense) matrix. The equivalent electrical distance between nodes $k$ and $l$ is thus given by the magnitude of the relevant entry of the $Z_{\text {bus }}$ matrix. Smaller $\left|Z_{k l}^{\text {bus }}\right|$ corresponds to shorter electrical distances.

That being said, based on the electrical distance between the target DERs systems and the point where the optimization criteria are applied, effectiveness (weighting factor) of the corresponding DERs could be calculated. DERs located at nodes with high electrical distance from the point of interest are expected to have less contribution to voltage correction or energy balance than similar agents located at closer (less electrical distance) nodes. That is simply called "effectiveness."

(3) Voltage Sensitivity. It was discovered that some nodes along the feeder could be susceptible to voltage problems due to increased penetration of the DERs. On the other hand, some nodes, mostly close to the stronger body of the feeder, are more robust and stable in similar situations. From the 
utility point of view, those robust nodes could be the best candidates for being DERs "hubs." That said, those nodes can tolerate large amount of power injection during high productivity and low consumption periods. To find the most suitable nodes to install large size DERs, like utility scale PV farms, such electrical hubs should be detected. To locate the so-called "electrical hubs" similar feeder simulations described earlier must be performed.

Results of this study could be utilized to address the conflicts between any global (feeder level) and many local (neighborhood level) optimization targets and to eliminate the necessity for high speed transmission of large chunks of data between the utility and the DERs.

Several cases were defined and simulated to demonstrate that, in presence of adequate information and processing power, it is readily achievable to satisfy a localized optimum solution by coordination of several DERs. By designing an efficient method of extracting required information from the local parameters, such as voltage and load in the neighboring agents, a global optimized solution would be reachable if a limited amount of data such as price, total load, or target load is transmitted by a central controller (utility company or grid operator).

\section{Conflict of Interests}

The authors declare that there is no conflict of interests regarding the publication of this paper.

\section{Acknowledgments}

This paper is based upon work supported partially by the Department of Energy under Award no. DE-OE0000230 and EPRI P.A. EP P32412 C15054. The authors gratefully acknowledge Nicholas Heine, Feng Cheng, Brian Arellano, Jon Hawkins, and Steve Willard for their valuable contributions and efforts.

\section{References}

[1] S. Burns and G. E. Gross, "Value of service reliability," IEEE Transactions on Power Systems, vol. 5, no. 3, pp. 825-834, 1990.

[2] R. Lasseter, A. Akhil, C. Marnay et al., The Certs Microgrid Concept, White Paper for Transmission Reliability Program, Office of Power Technologies, US Department of Energy, Washington, DC, USA, 2002.

[3] F. Cheng, S. Willard, J. Hawkins, B. Arellano, O. Lavrova, and A. Mammoli, "Applying battery energy storage to enhance the benefits of photovoltaics," in Proceedings of the IEEE Energytech, pp. 1-5, Cleveland, Ohio, USA, May 2012.

[4] C. Abbey, J. Robinson, and G. Joós, "Integrating renewable energy sources and storage into isolated diesel generator supplied electric power systems," in Proceedings of the 13th International Power Electronics and Motion Control Conference (EPEPEMC '08), pp. 2178-2183, IEEE, Poznań, Poland, September 2008.

[5] J. P. Barton and D. G. Infield, "Energy storage and its use with intermittent renewable energy," IEEE Transactions on Energy Conversion, vol. 19, no. 2, pp. 441-448, 2004.
[6] J. Eyer and G. Corey, "Energy storage for the electricity grid: benefits and market potential assessment guide," Tech. Rep., Sandia National Laboratory, 2010.

[7] J. Kumagai, "Swiss warehouse helps buffer the grid," IEEE Spectrum, no. 6, pp. 17-18, 2013.

[8] Itron Inc. Consulting and Analysis Service, "Public service new mexico electric energy efficiency potential study," chapter 3, Data Development and Economic Inputs, 2006, http://www.swenergy.org/news/news/documents/file/pnm_ electric_potential_study.pdf.

[9] A. Mammoli, C. Birk Jones, H. Barsun, D. Dreisigmeyer, G. Goddard, and O. Lavrova, "Distributed control strategies for high-penetration commercial-building- scale thermal storage," in Proceedings of the IEEE PES Transmission and Distribution Conference and Exposition (T and D '12), pp. 1-7, Orlando, Fla, USA, May 2012.

[10] J. Johnson, A. Ellis, A. Denda et al., "Pv output smoothing using a battery and natural gas engine-generator," Tech. Rep. SAND2013-1603, Sandia National Laboratories, Livermore, Calif, USA, 2013.

[11] http://www.gridlabd.org.

[12] S. Abdollahy, O. Lavrova, A. Mammoli, S. Willard, and B. Arellano, "PNM smart grid demonstration project from modeling to demonstration," in Proceedings of the IEEE PES Innovative Smart Grid Technologies (ISGT '12), pp. 1-6, Washington, DC, USA, January 2012.

[13] "Impact of high-penetration PV on d istribution system performance: example cases and analysis approach," Tech. Rep., EPRI, Palo Alto, Calif, USA, 2011.

[14] F. Cheng, S. Willard, J. Hawkins, B. Arellano, O. Lavrova, and A. Mammoli, "Applying battery energy storage to enhance the benefits of photovoltaics," in IEEE Energytech, pp. 1-5, May 2012.

[15] https://share.pnmresources.com/Public/Pages/default.aspx.

[16] A. E. Curtright and J. Apt, "The character of power output from utility-scale photovoltaic systems," Progress in Photovoltaics: Research and Applications, vol. 16, no. 3, pp. 241-247, 2008.

[17] A. Mammoli, M. Stadler, N. DeForest, H. Barsun, R. Burnett, and C. Marnay, "Software-as-a-service optimised scheduling of a solar-assisted hvac system with thermal storage," in Proceedings, 2nd International Conference in Microgeneration and Related Technologies, 2013.

[18] J. E. C. Sanchez, A complex network approach to analyzing the structure and dynamics of power grids [Diploma thesis], The Graduate College of The University of Vermont, October 2009. 


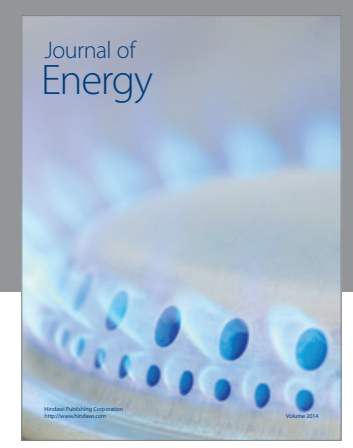

Journal of

Industrial Engineering
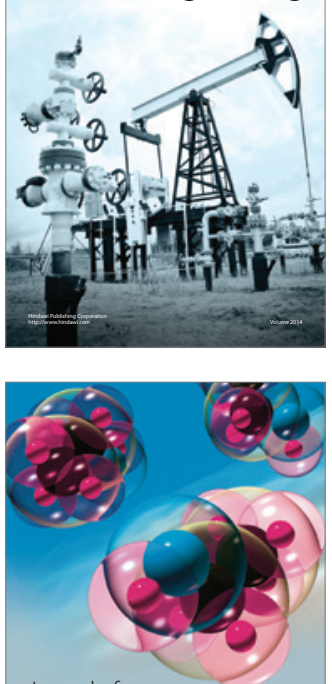

Fuels
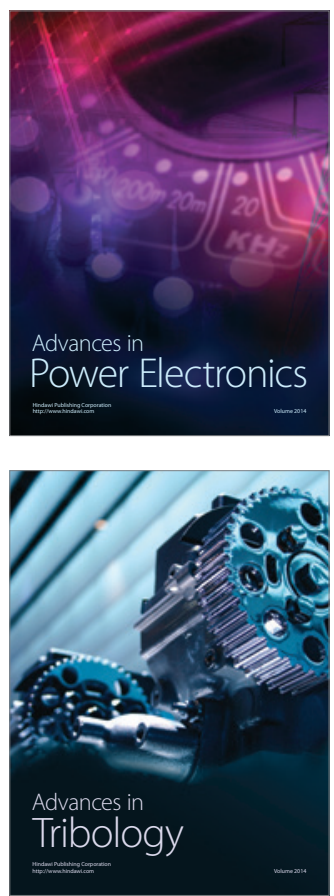

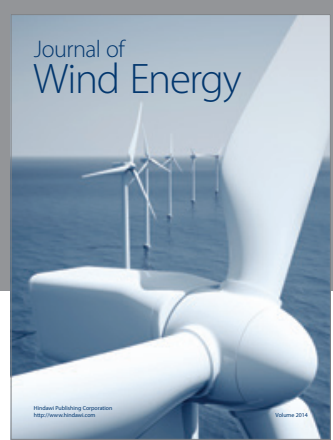

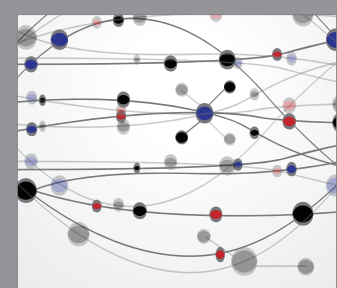

The Scientific World Journal

Submit your manuscripts at http://www.hindawi.com

Journal of

Structures
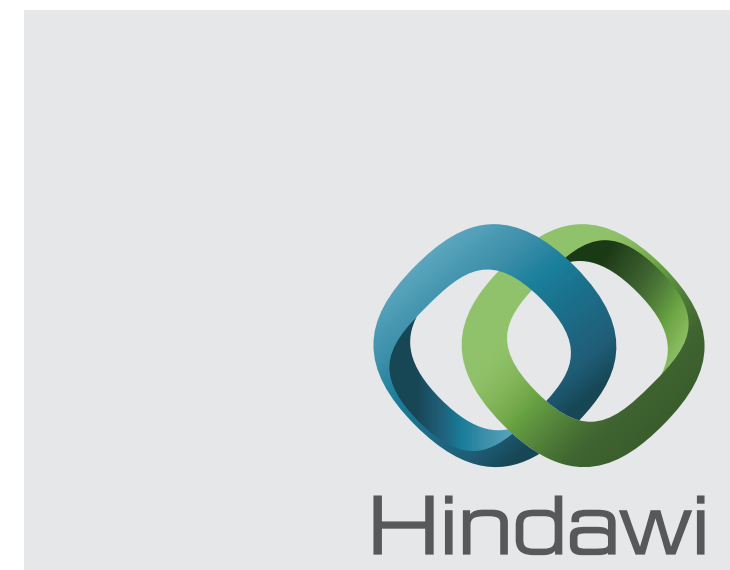

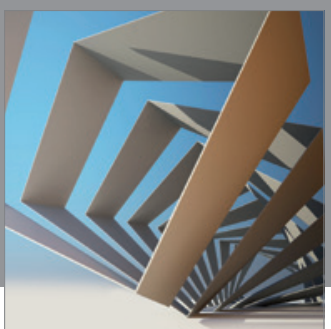

Rotating

Machinery
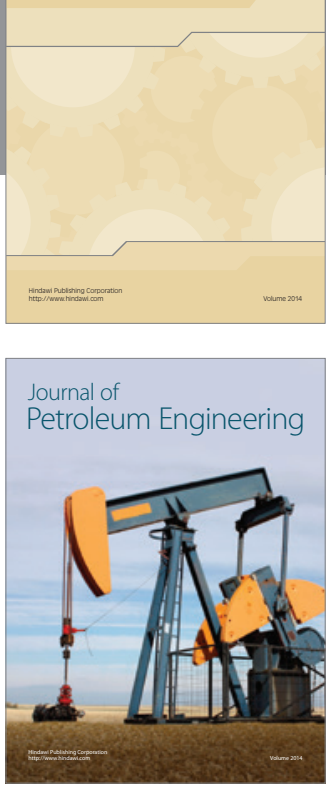

Journal of

Solar Energy
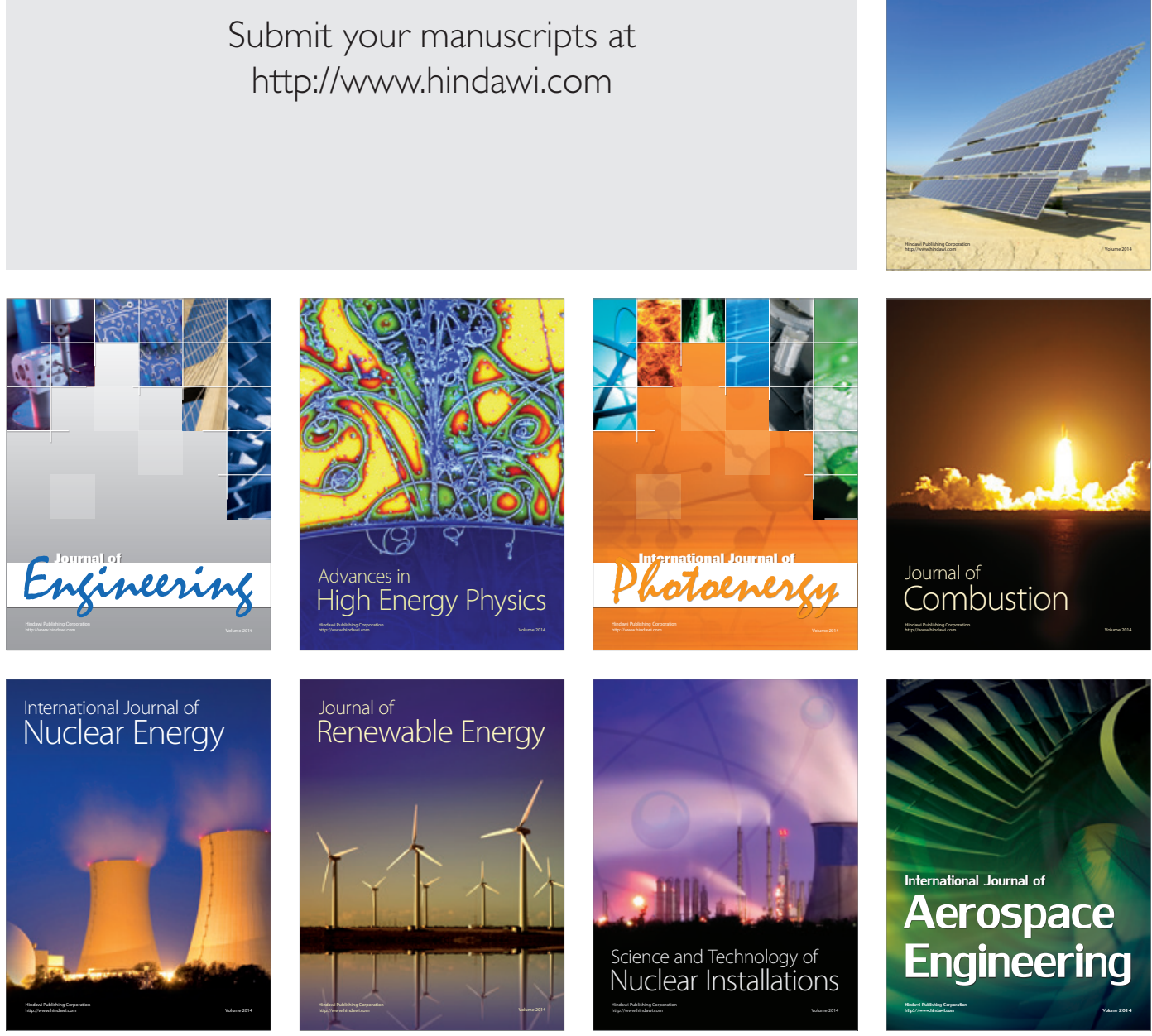